\title{
Tonal prediction of a faulty axial fan
}

\author{
Majdi Abid ${ }^{\mathrm{a}}$, Hassen Trabelsi ${ }^{\mathrm{a}}$, Mohamed Taktak ${ }^{\mathrm{a}, *}$, Jérôme Antoni ${ }^{\mathrm{b}}$, Jean Michel Ville ${ }^{\mathrm{b}}$, \\ Tahar Fakhfakh ${ }^{\text {a }}$, Mohamed Haddar ${ }^{a}$ \\ a Unité de Dynamique des Systèmes Mécaniques (UDSM), Ecole Nationale d'Ingénieurs de Sfax, Université de Sfax, BP N 1173-3038, Sfax, Tunisia \\ ${ }^{\mathrm{b}}$ Laboratoire Roberval UMR UTC - CNRS n 6253, Université de Technologie de Compiègne, BP 20529, F60205 Compiègne Cedex, France
}

\begin{abstract}
The current techniques for monitoring and diagnostics of rotating machines to their conditional predictive maintenance mainly are based on vibration monitoring. In this research, experimental and theoretical methods to predict the tonal noise of a faulty fan are developed and validated. These methods can be used on identifying defects of an axial fan based on an acoustic monitoring in situations where it is necessary to operate remotely due to the inaccessibility of the areas of event defects.
\end{abstract}

\section{Introduction}

Tonal noise emitted by rotating machine with blades like compressor, axial fan and centrifugal fan is one of the most important sources of noise in machinery. We can find fan in many applications: air conditioning system, car engine, turbo reactor engine, etc. Its tonal noise is emerged from the spectra of these systems and can be an undesirable source of sound. This component of sound can be amplified when the fan is faulty: for example a broken blade can change completely the spectra of sound of these machines, or an obstacle in front of its aspiration mouth can considerably rise the sound level. For this, and since many years, a lot of studies were interested to the prediction of sound generated by fan in their environment. Generally, fan can be used in duct or in free space. These models were interested on different types of faultless fan, but no one to a faulty case. One of the first models was developed by Gutin [1] in 1936 which considered the sound produced by a rotating constant force exerted by the blade on its medium. Lowson [2,3] extended the work of Lighthill [4] to establish a model of the sound generated by singularities in motion produced by the blade in their rotary motion. This work was followed by Fowks Williams and Hawkings [5] and they established their equation witch take in count the interaction between the

\footnotetext{
* Corresponding author.

E-mail addresses: mohamed.taktak.tn@gmail.com, Mohamed.Taktak@fss.rnu.tn (M. Taktak).
}

medium and a solid boundary. Another approach is to consider the fan disc swept by the blades like a distribution of dipole source exited by the passage of every blade. Morse and Ingard [6] obtained with this method an equation similar to the one obtained by Lowson $[2,3]$.

The objective of the present paper is the development of, first, a simple model of the tonal noise of an axial flow fan without and with defects. The second objective is the development of an experimental procedure to measure this noise. These two developed tools can be used in a technique of diagnostic of axial fan.

In this paper and after having presented the theoretical formulation to determine the acoustic noise radiated from an axial fan with and without defects in Section 2, the experimental procedure to measure this noise is presented in Section 3. In this section also, the theoretical model was validated by comparison with experimental results. Finally, theoretical and experimental results are presented and discussed in Section 5.

\section{Theoretical formulation}

In this section a model for fan noise prediction is derived, this model is used to calculate the acoustic pressure emitted by the fan at any point in space. Parameters of the fan like hub radius, blade tip radius, power of the motor, rotating speed and aerodynamics forces, are the inputs of this model. Our starting point is Ffowks Williams-Hawkings equation which is the summation of three terms relative to different types of sources: 
$\frac{\partial^{2} \rho}{\partial t^{2}}-c^{2} \Delta \rho=\frac{\partial q}{\partial t}-\frac{\partial F_{i}}{\partial x_{i}}+\frac{\partial^{2} T_{i j}}{\partial x_{i} \partial x_{j}}$

$\frac{\partial^{2} T_{i j}}{\partial x_{i} \partial x_{j}}$ is the quadripolar source term associated only to flow, $\frac{\partial F_{i}}{\partial x_{i}}$ is the dipolar source term, called charge noise caused by the interaction between solid and medium and $\frac{\partial q}{\partial t}$ is the monopolar source, results on the displacement of the medium by the blade in rotation.

In our study, we consider a well designed fan with thin blades and sharp edge. In the case of low mach number on blade tip, only the second term can be an efficient source of noise as mentioned in Gérard [7]. The other sources can be important for example in case of helicopter rotor or supersonic rotor etc.

By applying the Green function in free field on the second ter$\mathrm{m}$ of Eq. (1), the acoustic pressure is given as follow Khelladi et al. [8]:

$p(t)=-\frac{1}{4 \pi} \iint \frac{r_{i}}{c_{0} D o p r^{2}} \frac{\partial}{\partial \tau}\left(\frac{F_{i}}{D o p}\right) d S$

with $t$ is the time relative to the observer, $\tau$ is the time relative to the source, $F_{i}$ the component of the force $\vec{F}$ exerted by the blade on the medium, $r_{i}$ is the component of the source-observer vector $\vec{r}$ with $r=|\vec{r}|$, Dop $=1-\frac{\vec{r}}{r} \vec{M}$ is the Doppler factor. $\vec{M}=\frac{\vec{V}}{C_{0}}$ and $\vec{V}$ is the source speed.

After application of the Fourier transform and a variable change and a partial integration we get:

$P_{s}=\frac{i s \omega^{2}}{8 \pi^{2} c_{0}} \iint\left[\int_{0}^{\frac{2 \pi}{\omega}} \frac{r_{i} F_{i}}{r^{2}} e^{i s \omega\left(\tau+\frac{r}{c_{0}}\right)} d \tau\right] d S$

This equation allows the computation of the sth Fourier coefficient of the pressure sound in an observation point in free space due to a force distribution over the blade. As presented in Lowson [3] and Bridelance [9], the distributed force can be replaced by an equivalent one concentrated at the aerodynamic centre of the blade located approximately at $r_{\mathrm{s}}$ defined as:

$r_{s}=\frac{2}{3} \frac{\left(R 2^{3}-R 1^{3}\right)}{\left(R 2^{2}-R 1^{2}\right)}$

with $R_{1}$ and $R_{2}$ are respectively the hub and the blade tip radius.

Thus, we can eliminate the integral surface over the blade and Eq. (3) became:

$P_{s}=\frac{i s \omega^{2}}{8 \pi^{2} c_{0}} \int_{0}^{\frac{2 \pi}{\omega}} \frac{r_{i} F_{i}}{r^{2}} e^{i s \omega\left(\tau+\frac{r}{c_{0}}\right)} d \tau$

To obtain the final form of this model we replace $\vec{r}$ and $\vec{F}$ by their component described in Fig. 1:

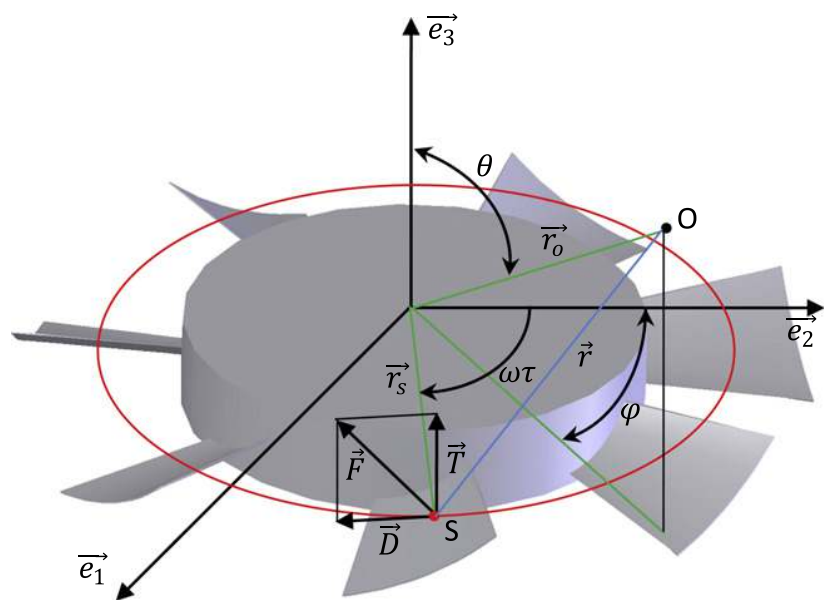

Fig. 1. Coordinate system.

$$
\begin{aligned}
& \vec{r}=\left(\begin{array}{c}
r_{0} \sin \theta \sin \varphi-r_{S} \sin (\omega \tau) \\
r_{0} \sin \theta \cos \varphi-r_{s} \cos (\omega \tau) \\
r_{0} \cos \theta
\end{array}\right) \\
& \vec{F}=\left(\begin{array}{l}
F_{1} \\
F_{2} \\
F_{3}
\end{array}\right)=\left(\begin{array}{c}
D \cos (\omega \tau) \\
-D \sin (\omega \tau) \\
T
\end{array}\right) \\
& \|\vec{r}\|=\sqrt{r_{0}^{2}+r_{s}^{2}-2 r_{0} r_{s} \sin \theta \cos (\omega \tau-\varphi)}
\end{aligned}
$$

In the case of far field we have $r_{Q}>>r_{s}$ and Eq. (8) became:

$\|\vec{r}\| \approx r_{o}-r_{s} \sin \theta \cos (\omega \tau-\varphi)$

By replacing Eqs. (6)-(8) in Eq. (5) the Fourier component of the acoustic pressure due to the aerodynamic force concentrated at $S$, at the observer position $O$ is given as follow:

$$
\begin{aligned}
P_{s}= & \frac{i s \omega^{2}}{8 \pi^{2} c_{0} r_{0}} e^{i s \omega \frac{r_{0}}{c_{0}}} \\
& \times \int_{0}^{\frac{2 \pi}{\omega}}\left([T \cos \theta-D \sin \theta \sin (\omega \tau-\varphi)] e^{i s \omega \tau} e^{-i s \omega \sin \theta \frac{r_{s}}{c_{0}} \cos (\omega \tau-\varphi)}\right) d \tau
\end{aligned}
$$

The relation of Jacobi-Anger and its derivative are expressed as follow:

$e^{-i A \cos \alpha}=\sum_{q=-\infty}^{+\infty}(-i)^{q} J_{q}(A) e^{-i q \alpha}$

$\sin \alpha e^{-i A \cos \alpha}=-\frac{1}{A} \sum_{q=-\infty}^{+\infty} q(-i)^{q} J_{q}(A) e^{-i q \alpha}$

By using expression (11) and (12) and after simplification Eq. (10) becomes:

$P_{s}=\frac{i s \omega}{4 \pi c_{0} r_{0}} e^{i s \omega \frac{r_{0}}{c_{0}}} \sum_{q=-\infty}^{+\infty}(-i)^{q} J_{q}\left(s \omega \sin \theta \frac{r_{s}}{c_{0}}\right) e^{i q \varphi}\left(\cos \theta T_{s-q}+\frac{q c_{0}}{s \omega r_{s}} D_{s-q}\right)$

$T$ and $D$ are respectively trust and drag (Fig. 2) and $T_{s-q}$ and $D_{s-q}$ are their $s-q$ th Fourier coefficient. These two components can be related by a coefficient which is related to the blade pitch.

According to Fig. 2 we can write $T=\frac{D}{\tan \alpha}$ with $\alpha=30^{\circ}$ then $T=2 . D$.

The drag force is directly related to the torque applied to the $\mathrm{r}$ adius $r_{s}\left(C=D r_{s}\right)$. Also, The torque is related to the power and angular frequency $(C=p / \omega)$. If we have $B$ blades then:

$D=\frac{P}{B \omega r_{s}}$

The sound production in a fan is mainly due to the aerodynamic force variation. In fact a constant aerodynamic force is inefficient compare to one how vary in its movement. The variation is caused by a non-uniform flow at the suction mouth caused by an obstacle

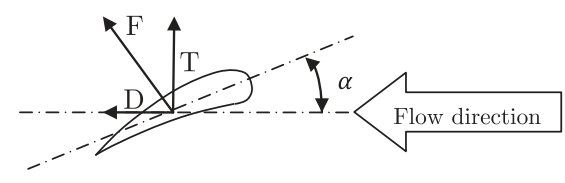

Fig. 2. Aerodynamics force. 


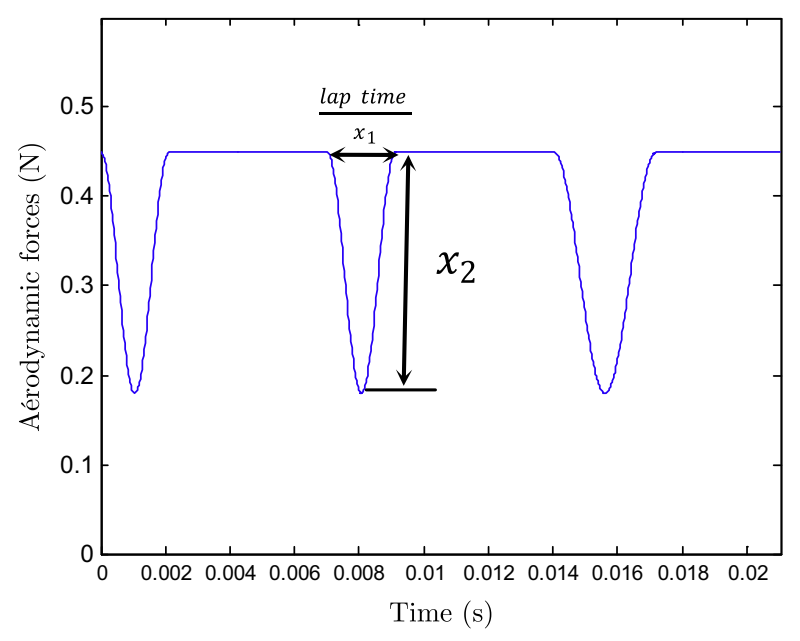

Fig. 3. Aerodynamics force variation over a revolution.

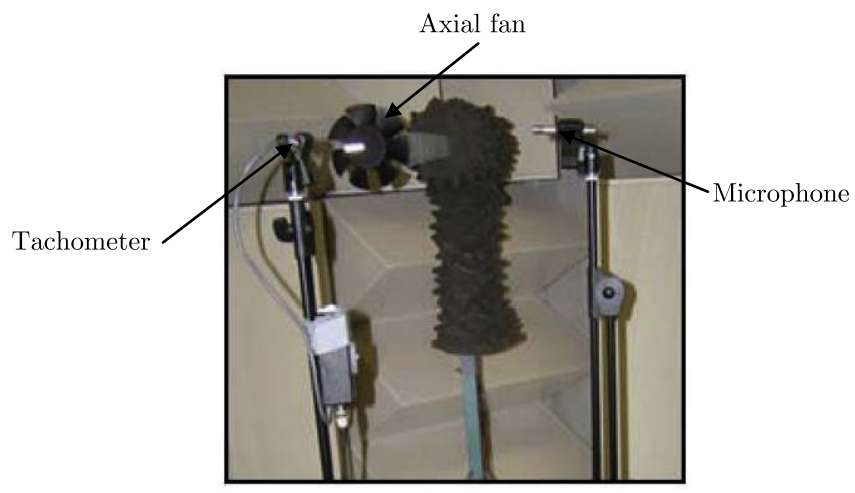

Fig. 4. Experimental materials.

in this flow. In our case struts how maintains the motor cause the variation of the loading forces periodically as presented by Boltezar et al. [10]. Fig. 3 shows the periodic form of the trust force adopted.

The third drop is larger than the other since the third struts is 1.5 larger than the other as it contains wire for the motor supply.

The Eq. (13) is used for a singular aerodynamic force. In a fan with $B$ blades we have $B$ forces. In the observer position we obtain the superposition of the sound produced by the $B$ forces.

$P_{s}=P_{s}^{1}+P_{s}^{2}+\ldots+P_{s}^{B}$

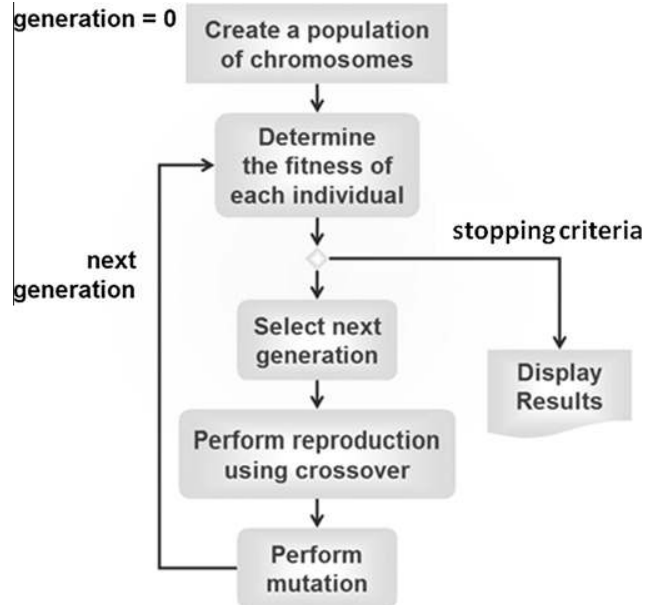

Fig. 6. Steps in the genetic algorithm.

The sound received at the observer position from one blade is shifted in time compared to the sound received from the following blade. This time is related to the fan design. The time lag between two successive blades is:

$t_{d}=\frac{2 \pi}{B \omega}$

Then we can relate the Fourier coefficient of each blade force to each other. If we chose $P_{s}^{1}$ as a reference we can write:

$P_{s}=P_{s}^{1}\left(1+e^{i s \omega t_{d}}+e^{i s \omega 2 t_{d}}+\ldots+e^{i s \omega B t_{d}}\right)$

The quantities between bracket is related to the fan shape, with this term we can simulate a broken blade if we delete one term of the summation, we can also simulate an uneven blade spacing configuration if we change the time lag $t_{d}$.

\section{Experimental validation}

\subsection{Experiment setup}

The experiences were conducted in the anechoic chamber of the University of Technology of Compiegne. A 0.5 in microphone was placed in the observer position and a tachometer is placed in front of the fan (Fig. 4). These two signals are sampled together using a sampling rate of $16 \mathrm{KHz}$.

The pulse signal of the tachometer allows extracting the tonal noise of the fan and eliminating the broad band noise. In fact the tachometer signal was used to resembling the raw signal which

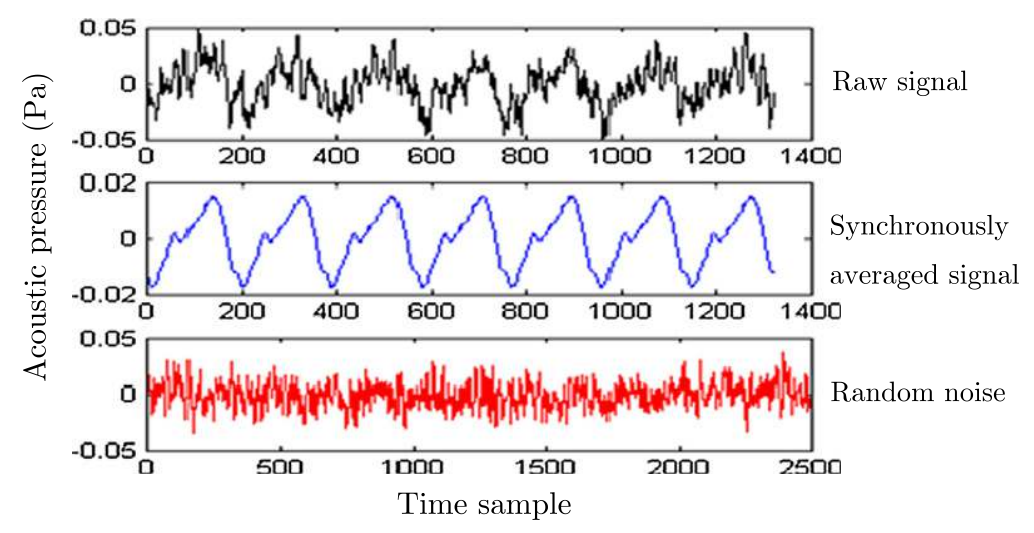

Fig. 5. Steps in signal treatment. 


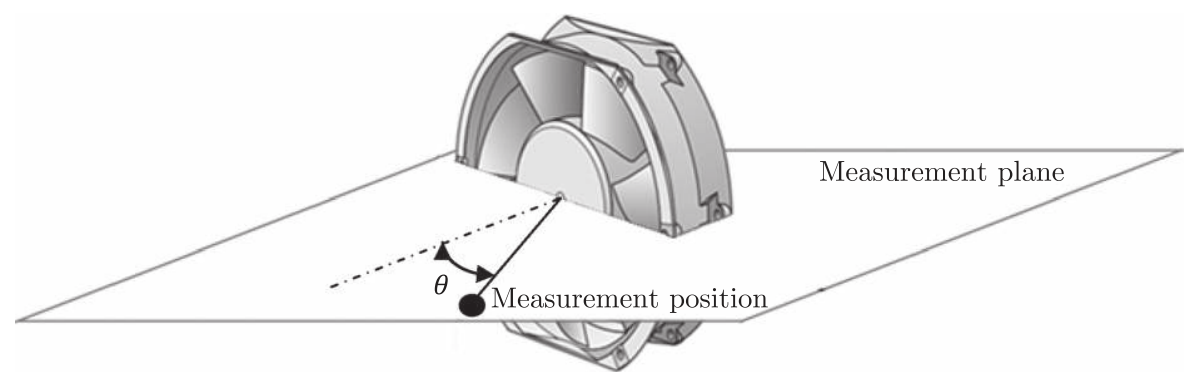

Fig. 7. Measurement plane.

Table 1

Experimental conditions.

\begin{tabular}{ll}
\hline Fan speed & $2852 \mathrm{RPM}$ \\
Radius of the hub & $37 \mathrm{~mm}$ \\
Blade tip radius & $70 \mathrm{~mm}$ \\
Number of blades & 7 \\
Motor power & $26 \mathrm{~W}$ \\
\hline
\end{tabular}

Table 2

Results of optimization.

\begin{tabular}{ll}
\hline$x_{1}$ & $x_{2}$ \\
\hline 3.233 & $36.75 \%$ of the maximum value of $\|\vec{F}\|$ \\
\hline
\end{tabular}

is time-based signal to a rotary-based signal this overcomes the rotation speed variation caused by the motor power supply variation, then the resembled signal is averaged over many periods. This synchronously averaged signal is the rotary sound, the spectra of this signal is the tonal noise at the blade passage frequency (Fig. 5). This technique is order analysis.

\subsection{Model calibration}

To compare theoretical result to the experimental one we have to define all parameter of this model. All quantities in Eq. (13) are known except $T_{s-q}$ and $D_{s-q}$ which depend on $x_{1}$ and $x_{2}$. These two parameters were estimated by comparing the calculated and measured spectra of the fan noise. This amounts to find the couple $\left(x_{1}, x_{2}\right)$ that minimize the difference between theoretical and experimental spectra. To achieve this goal, the genetic algorithm is chosen to find this minimum.
In fact the genetic algorithm, that the algorithm is presented in Fig. 6, can find the minimum of a complex parametric problem. It is an evolutionary algorithms (EAs), which generate solutions to optimization (minimization) problems using techniques inspired by natural evolution, such as inheritance, mutation, selection, and crossover. We have to define a fitness function which is the least square difference between experimental and calculated spectra.

In our case each chromosome is a combination of two genes $\left(x_{1}\right.$, $x_{2}$ ), the stopping criteria was fixed to 5000 generations.

The reference position is arbitrary chosen and it is situated at $0.55 \mathrm{~m}$ from the centre of the fan and makes an angle of $40^{\circ}$ with its axe (Fig. 7).

The experimental conditions are regrouped in Table 1:

The genetic algorithm toolbox of MATLAB is used to determine $x_{1}$ and $x_{2}$. The coast function is the sum of squared residuals; witch is defined as the difference between the value predicted by the model and the experimental value at a selected order. After 5000 generation the results are regrouped in Table 2.

The values of $x_{1}$ and $x_{2}$ are then used for all the following simulation. In Fig. 8 the theoretical and experimental spectra for the reference position are compared. A good agreement between the two spectra is observed especially for the three first order $(7,14$, 21).

\subsection{Model validation: directivities experiences}

The acoustic pressure was measured on a circle of radius $0.55 \mathrm{~m}$. The centre of the measuring circle is the centre of the fan. And the measurement plan contains the fan's axe (Fig. 7). The theoretical and experimental directivities are compared at orders which are multiple of the number of blade.

Fig. 9 presents the directivities at orders 7, 14 and 21 for a flawless fan. The respective frequencies for these orders are $332.7 \mathrm{~Hz}$, $665.4 \mathrm{~Hz}$ and $998.2 \mathrm{~Hz}$. This figure shows the dipolar form of the directivity according the direction $0^{\circ}-180^{\circ}$ for several orders. This
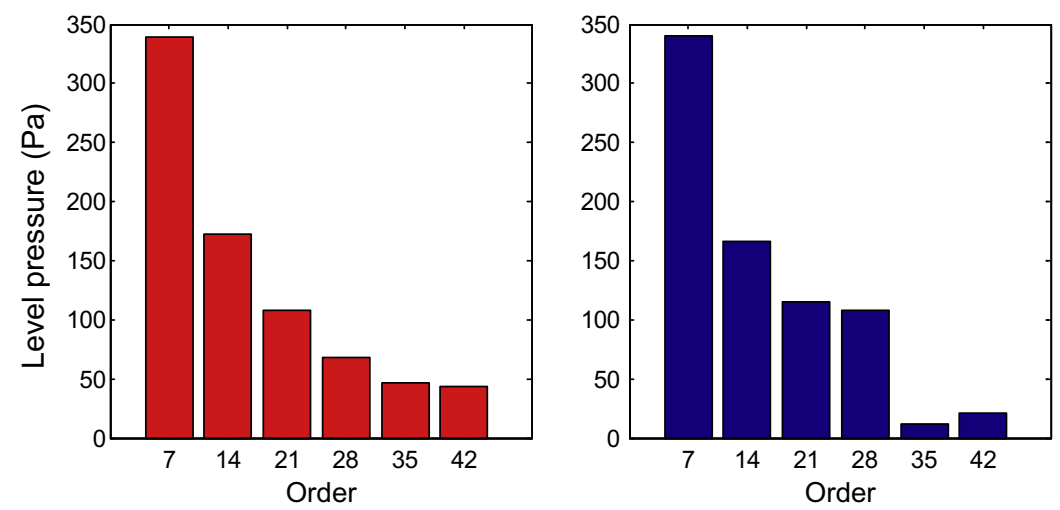

Fig. 8. Compared theoretical and experimental spectra for reference position. 

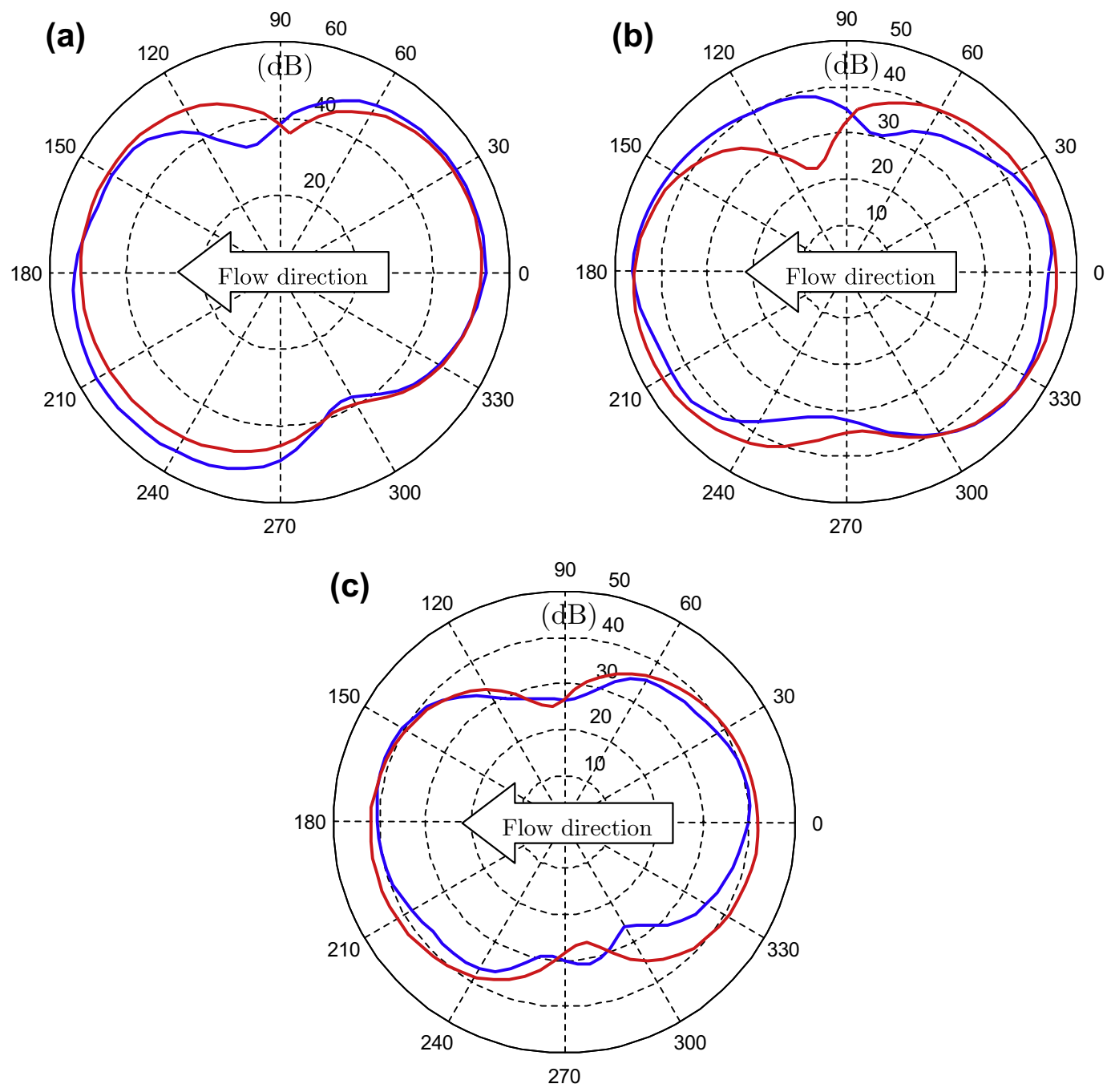

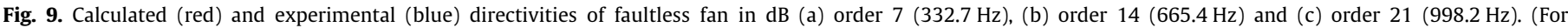
interpretation of the references to color in this figure legend, the reader is referred to the web version of this article.)

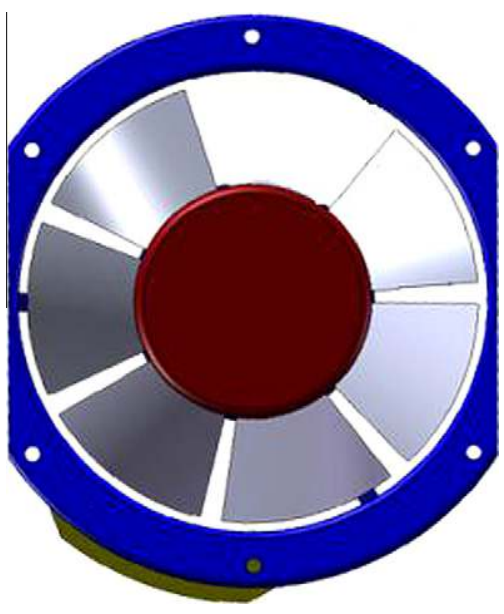

Fig. 10. Faulty fan: broken blade.

figure shows a good agreement between theoretical and experimental directivities: the values of computed and measured pressures are close; in fact although some gaps, the average error is still acceptable: for the order 7 the average error is about $5.5 \%$ this percentage became $7.9 \%$ for order 14 and $8.1 \%$ for order 21 which are admissible. There is a slight rotation between the two directivities. Differences in values can be explained by some loss in the experiments.

\section{Faulty fan directivities}

\subsection{Faulty fan with broken blade}

After the validation of the model in the case of faultless fan, in this section the faulty case is studied. Several defects may appear on a fan. In a first step, we studied a totally broken blade; an object in the suction mouth can cause disturbing noise. The blade can be also deviated after a choc but it cannot happen in our case since we have a plastic fan.

In the following comparison we have considered a fan with a totally broken blade. In this case the spectra of the fan is not concentrated only at order multiple of the number of blades, the spectra is spread over the entire frequency range all order are exited. In fact now we have the phenomenon of the void left by the broken blade that is repeated every rotation.

The point force formulation is a suitable for predicting the tonal noise emitted by a broken blade fan. Fig. 11 shows that this 

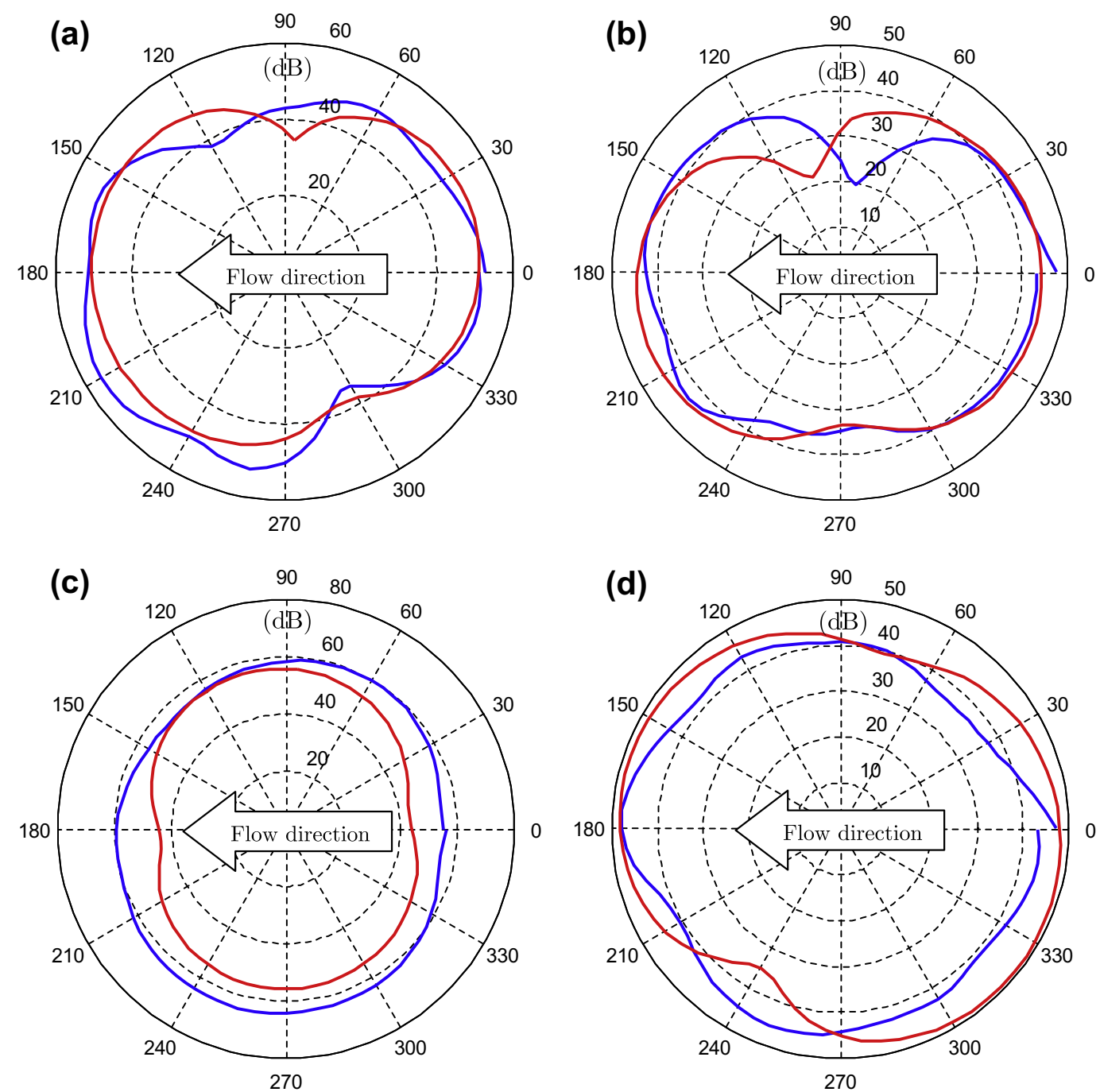

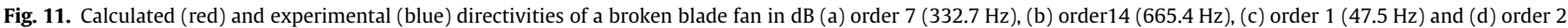
$(95 \mathrm{~Hz})$. (For interpretation of the references to color in this figure legend, the reader is referred to the web version of this article.)
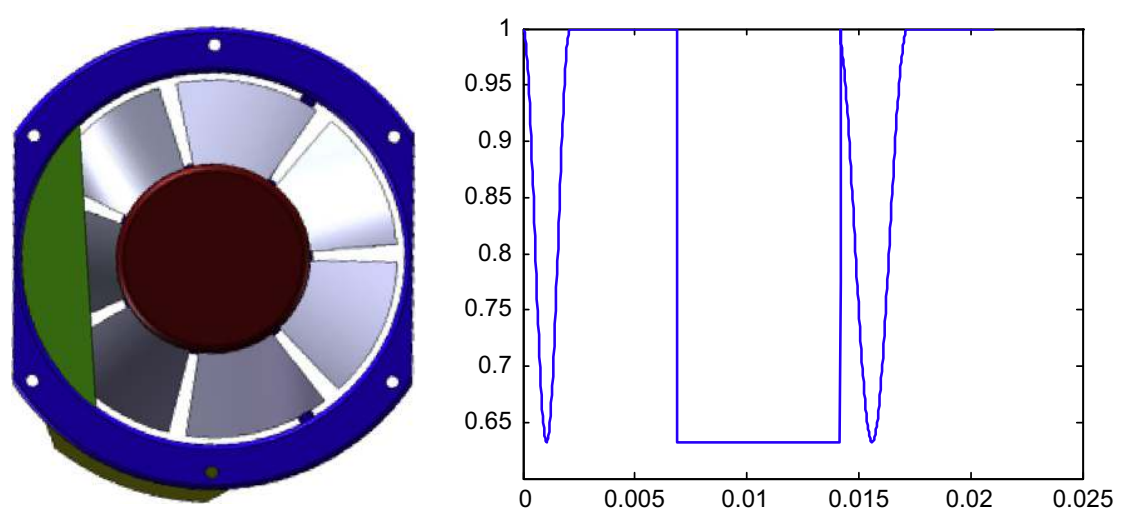

Fig. 12. Faulty fan: clogged mouth suction and its corresponding aerodynamic force.

model can fit the variation of directivities. For Fig. 10c a lag of $10 \mathrm{~dB}$ can be noticed. The shape of the directivities is successfully predicted despite some large shift at some angle. This is due to the experimental condition or the inefficiency of the model at low frequency. The appearance of order multiple of the rotation frequency can be regarded as a criterion for judging on the presence or absence of a default since the power issued at these order$\mathrm{s}$ is negligible for a flawless fan.

\subsection{Faulty fan with clogged mouth suction}

The next simulated default is partially clogged mouth suction (Fig. 12). For this the shape of the aerodynamic force $\vec{F}$ must be changed. A rectangular window with the same width of the obstacle is applied to the shape described in figure (variation force).

Although the good agreement at the order 7 calculated directivities at order 14 and the other order $1,2 \ldots$ are not consistent 

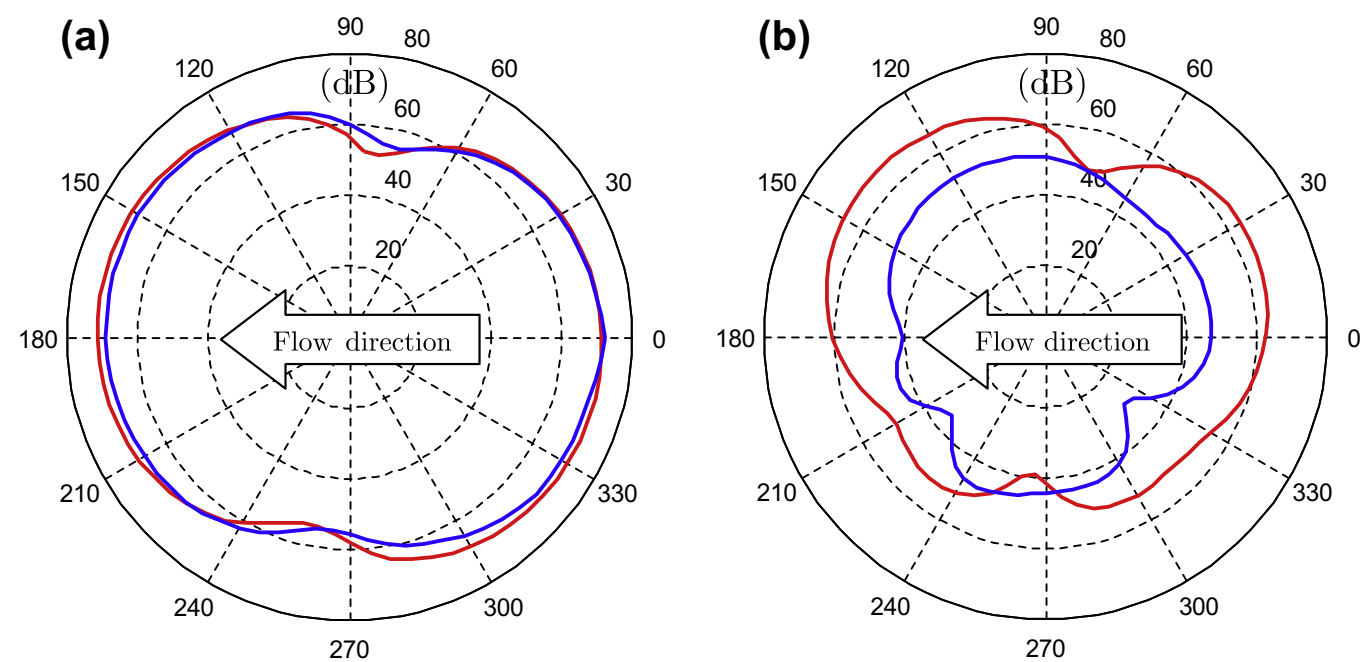

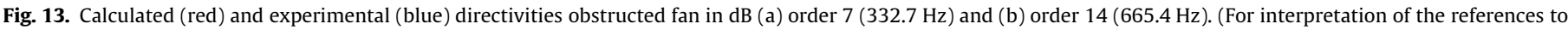
color in this figure legend, the reader is referred to the web version of this article.).

with the experiment one as presented in Fig. 13. The shape of the directivities is globally well estimated but the large gap between experience and theoretical directivities must be improved. For this other shape of aerodynamics forces must be tested.

From these two faulty fan simulations some criteria can be considered to estimate the presence or not of default on the fan: the first one is the appearance of order which is multiple of the rotating frequency not the blade passing frequency, the second one is the change of the shape of the directivities, these two criteria are qualitative. We can consider also the amplification of the sound radiated at a chosen order for example for a nonfaculty fan at order 7 the acoustic pressure is around $50 \mathrm{~dB}$ but for the clogged one is around $70 \mathrm{~dB}$.

\section{Conclusion}

In this work a simple model was adopted to predict the tonal noise of an axial fan. Experiences have been performed in two cases: with a faultless fan and with a faulty one. Good agreement between experience results and simulations has been obtained. The point force formulation is a fast and simple method to predict fan noise. But it is not suitable at higher frequency (high order) and unable to detect a motor default or bearing failure. This model can be a tool to concept a more quite fan or can be used in an inverse problem to localize the source noise on a fan's blade.

\section{Acknowledgements}

This work was carried out in the Framework of the TunisianFrench Research Project CMCU 08G1140 about Monitoring and diagnosis of Rotating Machines, Improved isolation and the sound Environmental Impact.

\section{References}

[1] Gutin L. On the sound field of a rotating propeller translated in 1948 from physikalische zeitschrift der sowjetunion. NACA TM 1195; 1936.

[2] Lowson MV. The sound field for singularities in motion. Proc Royal Soc London 1965;A286:559-72.

[3] Lowson MV. Theoritical analysis of compressor noise. J Acoust Soc Am 1970;47:371-85.

[4] Lighthill MJ. On sound generated aerodynamically. Proc Royal Soc London 1952;A211:564-87.

[5] Fowcs Williams JE, Hawkings DL. Sound generation by turbulence and by surfaces in arbitrary motion. Philos Trans Royal Soc London 1969;264:321-42.

[6] Morse M, Ingard KU. Theoritical acoustics. McGraw-Hill, Inc.; 1986.

[7] Gérard A. Bruit de raie des ventilateurs axiaux: estimation des sources aéroacoustiques par modèles inverses et méthode de contrôle. Thesis, Université de Poitiers; 2006.

[8] Khelladi S, Kouidri S, Bakir F, Rey R. Predicting tonal noise from a high rotational speed centrifugal fan. J Sound Vib 2008;313:113-33.

[9] Bridelance. Etude aéroacoustique des spectres de raies générés par les ventilateurs axiaux en régime subsonique. Thesis, Ecole Nationale Supérieure des Art et Metiers; 1982.

[10] Boltezar M, Mesaric M, Kuhelj A. The influence of uneven blade spacing on the SPL and noise spectra radiated from radial fans. J Sound Vib 1998;216(4):697-711. 